\title{
EVALUACIÓN TÉCNICO-VISUAL DE ESTRUCTURAS SEGÚN NEC-SE-RE EN EL SECTOR "LA ARMENIA 1" PARA LA DETERMINACIÓN DE RIESGO ANTE FENÓMENOS NATURALES ESPECÍFICOS
}

\section{TECHNICAL-VISUAL EVALUATION OF STRUCTURES ACCORDING TO NEC-SE-RE IN THE "ARMENIA 1" SECTOR, FOR THE DETERMINATION OF RISK TO SPECIFIC NATURAL PHENOMENA}

\author{
WILSON CANDO ${ }^{1}$ \\ ÓSCAR JARAMILLO ${ }^{2}$ \\ JORGE BUCHELI ${ }^{3}$ \\ XAVIER PAREDES ${ }^{4}$
}

Recibido: 18 de octubre de 2017

\footnotetext{
${ }^{1}$ Pontificia Universidad Católica del Ecuador, Facultad de Ingeniería, Quito, Ecuador (wocando@puce.edu.ec).

${ }_{2}^{2}$ Pontificia Universidad Católica del Ecuador, Facultad de Ingeniería, Quito, Ecuador (ojaramillo602@puce.edu.ec).

${ }_{3}^{3}$ Pontificia Universidad Católica del Ecuador, Facultad de Ingeniería, Quito, Ecuador (jabucheli@puce.edu.ec).

${ }^{4}$ Pontificia Universidad Católica del Ecuador, Facultad de Ingeniería, Quito, Ecuador (eparedes001@puce.edu.ec).
} 
Nㅔㅅㅣ 112 


\section{EVALUACIÓN TÉCNICO-VISUAL DE ESTRUCTURAS SEGÚN NEC-SE-RE EN EL SECTOR "LA ARMENIA 1" PARA LA DETERMINACIÓN DE RIESGO ANTE FENÓMENOS NATURALES ESPECÍFICOS}

\section{TECHNICAL-VISUAL EVALUATION OF STRUCTURES ACCORDING TO NEC-SE-RE IN THE "ARMENIA 1" SECTOR, FOR THE DETERMINATION OF RISK TO SPECIFIC NATURAL PHENOMENA}

Wilson Cando, Óscar Jaramillo, Jorge Bucheli, Xavier Paredes.

Keywords: Risk, Hazard, Vulnerability, La Armenia 1, Risk Matrix, FEMA P-154.

Palabras clave: Riesgo, Peligro, Vulnerabilidad, La Armenia 1, Matriz de Riesgos, FEMA P-154.

\section{RESUMEN}

En esta publicación se realiza una evaluación de riesgos ante fenómenos naturales para el sector "La Armenia 1", ubicada en el Valle de los Chillos, Quito, Ecuador.
El estudio se concentra principalmente en la evaluación de las estructuras ante riesgos sísmicos y volcánicos; pues son los fenómenos naturales que por la ubicación y características del sec- 
tor afectarían en mayor medida al mismo y tienen mayor probabilidad de ocurrencia como se lo ha visto a lo largo de los años.

Para la evaluación ante el riesgo sísmico se utilizó la metodología FEMA P-154, realizada por la Agencia Federal de Manejo de Emergencias (Federal Emergency Management Agency) de los Estados Unidos; misma que es sugerida por la norma ecuatoriana de la construcción en su capítulo de Peligro Sísmico y Diseño Sismo Resistente (NECSE-DS). Con dicha metodología, mediante una rápida evaluación visual se puede determinar el tipo de estructura y las irregularidades que presenta, para a continuación con el uso de un sistema de puntuación planteado por FEMA P-154 se podrá establecer la probabilidad de colapso de una estructura ante la ocurrencia del máximo sismo considerado en un período de retorno de 2475 años.

Para la evaluación ante el riesgo volcánico el presente trabajo se basó fundamentalmente en los estudios y publicaciones realizadas por el Instituto Geofísico de la Escuela Politécnica Nacional y la Secretaría de Gestión de Riesgos; y después de analizar la información recopilada se determinó que los fenómenos volcánicos que más afectarían al sector "La Armenia 1" serían la caída de ceniza y el flujo de lahares y escombros. Para estos fenómenos se presenta una matriz de riesgos que realiza una evaluación cualitativa de los efectos que pueden provocar.

\section{ABSTRACT}

This paper carries a risk evaluation fore natural phenomena in "La Armenia 1" placed in Valle de los Chillos, Quito, Ecuador.

This study focuses mainly in the structures evaluation against seismic and volcanic risks; those are natural phenomena that due to the location and properties of the neighborhood, affects it to a greater extent and are more likely to occur as it has been seen through the years.
For the evaluation of seismic risk, it was created the FEMA P-154, carried out by the Federal Emergency Management Agency of the United States; same that is suggested by the Ecuadorian Construction norm in its chapter of Seismic Hazard and Resistive Earthquake Design (NEC-SE-DS). Using this methodology, by means of a quick visual evaluation it is possible to determine the type of structure and irregularities that it presents, and then with the use of a ran- 
king system proposed by FEMA P-154 it will be possible to establish the probability of a structure collapse before occurs a maximum earthquake considered to return approximately every 2475 years.

For the evaluation of a volcanic risk, this paper was based fundamentally in different studies and publications realized by the Geophysical Institute of the
National Polytechnic School and the Risk Management Department; after analyzing the information collected, it resulted that volcanic phenomena that would most affects "La Armenia 1" would be the fall of ash and flow of lahars and debris. For these phenomena, a risk matrix is presented that makes a qualitative evaluation of the different effects that can be caused.

\section{PRESENTACIÓN DEL CASO}

Como consecuencia de los últimos acontecimientos en cuanto a fenómenos naturales que han amenazado al Distrito Metropolitano de Quito, como es: el sismo de 5.1 en la escala de Richter generado el 12 de agosto de 2015 (Cuatro temblores sacudieron a Quito, 2015), así como el sismo de $7.8 \mathrm{Mw}$ con epicentro entre las parroquias de Pedernales y Cojimíes ocurrido el 16 de abril de 2016 (IGEPN, 2016) que afectó a diversas zonas del país y las dos últimas erupciones importantes del volcán Cotopaxi registradas el 14 de agosto del 2015 (Chamorro, 2015), a partir de lo cual el volcán ha mantenido una actividad intermitente hasta el presente, es que se considera importante el estudio de ambos fenómenos naturales en el Valle de los Chillos, específicamente en el sector la Armenia 1 (Anexo 1), pertenecientes al Distrito Metropolitano de Quito.

El Valle de los Chillos se encuentra en una zona de alta actividad sísmica, es esta una zona de categoría $V$, según la norma ecuatoriana de la construcción en su capítulo NEC-SE-DS; de la misma manera con el reciente inicio de una fase eruptiva del volcán Cotopaxi se ha determinado que existe una gran cantidad de propiedades construidas en zonas de riesgo. El sector en estudio se vería afectado principalmente por los flujos laháricos que correrían por el cauce del río San Pedro, para la determinación del área de estudio se utilizó el mapa de amenazas del volcán Cotopaxi que se indica en (Secretaría de Gestión de Riesgos, s.f). 


\section{RIESGO SíSMICO}

\section{Metodología FEMA P-154}

El Análisis Visual Rápido conocido como (RVS), por sus siglas en inglés "Rapid Visual Screening", es un procedimiento que se ha desarrollado con la finalidad de identificar aquellas estructuras que son potencialmente peligrosas ante eventos sísmicos, es decir que frente a un evento sísmico son preocupantemente vulnerables. Una vez que se ha identificado a una estructura como potencialmente peligrosa, se la debe evaluar a mayor profundidad por un profesional con experiencia en el diseño sísmico, quien ayudará a determinar si en verdad la construcción es peligrosa sísmicamente (FEMA, 2015).

El procedimiento RVS usa una metodología que se basa en un estudio visual de la estructura, lo que implica la recopilación de datos de la misma en formularios específicos para esta metodología. La persona que conduce el estudio y recopilación de datos de la estructura realiza una observación de la misma desde el exterior de la construcción y de ser posible desde el interior.

El objetivo de los formularios es ayudar a determinar una puntuación final para cada estructura que se analice, lo cual nos proveerá una indicación de cuál sería el desempeño sísmico esperado de la estructura. Existen dos niveles de análisis: el Nivel 1,y adicional a este se tiene un nivel opcional, Nivel 2.

El formulario de Nivel 1 recolecta información de la descripción de la estructura que incluye: el uso de la misma, su tamaño, geometría, fotografía, boceto y otra documentación, así como datos pertinentes al desempeño sísmico de la estructura (FEMA, 2015). A cada estructura se le asigna una puntuación básica que está en función del sistema estructural que constituye la misma, la puntuación básica y los diversos sistemas estructurales que reconoce el FEMA se encuentran definidos en el formulario del Nivel 1.

El evaluador obtendrá la puntuación final afectando la puntuación básica con los modificadores de puntuación, los cuales están relacionados con los atributos de desempeño de la estructura que se puedan observar en campo, estos son sumados o restados a la puntuación básica para así obtener la puntuación final del Nivel 1.

Como ya se mencionó existe también un nivel opcional de análisis, este se define como nivel 2. El nivel 2 es un análisis más detallado del edificio, para ser realizado se utiliza el formulario correspondiente a este nivel, el cual permite al evaluador ajustar la calificación final de la estructura. 
La puntuación básica, modificadores de puntuación y puntuación final han sido desarrolladas tomando en cuenta la probabilidad del colapso de la estructura debido a la ocurrencia de un sismo poco frecuente (FEMA, 2015), es decir debido al movimiento de suelo que se produce como consecuencia del Máximo Sismo Considerado (MCE).

El rango en el cual oscila la puntuación final está entre 0 a 7, una mayor puntuación indica que la estructura tiene un mejor desempeño sísmico y una probabilidad baja de colapso. FEMA sugiere que a este rango de calificaciones se establezca un valor mínimo para diferenciar la respuesta de una estructura ante el MCE entre aceptable y no aceptable. Este valor mínimo se lo llama "CutOff" y se sugiere que sea 2; y representa el valor para el cual estructuras con esta o menor calificación tendrán que ser sometidas a evaluaciones más detalladas, pues no se espera una buena respuesta de la estructura (FEMA, 2015).

Un aspecto fundamental en la evaluación y calificación de la estructura es que la calificación de la misma depende de la región sísmica en donde se encuentra implantada la estructura, buscando determinar la probabilidad de colapso para el MCE, para lo cual FEMA P-154 ha definido cinco regiones sísmicas y un formulario específico para cada una de ellas: Regiones de Baja Sismicidad, Regiones de Moderada Sismicidad, Regiones de Moderadamente Alta Sismicidad, Regiones de Alta Sismicidad y Regiones de Muy Alta Sismicidad.

Para nuestro sector en estudio, al seguir lo que indica FEMA se estableció que corresponde a una zona de Muy Alta Sismicidad, lo cual se ha determinado verificando los valores de aceleración espectral a período corto "Ss" y a un segundo "S1" con los cuales mediante la (Tabla 1) se obtuvo la región sísmica. Los valores de SS y S1 fueron obtenidos con la aplicación del USGS "Worldwide Sismic Design Tool Beta" (Gráfico 1) y comprobados con las Curvas de Peligro Sísmico para Quito a diferentes Períodos Estructurales indicadas en (Peligro Sísmico Diseño Sismo Resistente NEC-SE-DS, 2015)

El formulario que se ha utilizado para el presente estudio se lo indica en el (Anexo 2). 
EVALUACIÓN TÉCNICO-VISUAL DE ESTRUCTURAS SEGÚN NEC-SE-RE EN EL SECTOR

"LA ARMENIA 1" PARA LA DETERMINACIÓN DE RIESGO ANTE FENÓMENOS NATURA|||||ln LES ESPECÍFICOS.

Tabla 1. Deicrminación de las Repiones Sismicas en hase a la Kepoeta de Aceleración Lapecinal parn MCE.

\begin{tabular}{|c|c|c|}
\hline \multirow[t]{2}{*}{ Reción Siamice } & \multirow{2}{*}{$\begin{array}{l}\text { Respuesta de Acelereción } \\
\text { tapectral, \$s (periodo } \\
\text { corte o } 0.2 \text { segundos) }\end{array}$} & \multirow{2}{*}{$\begin{array}{l}\text { Respuesta de Aceleracibon } \\
\text { Espectral, } 51 \text { (periodo } \\
\text { largo o } 1 \text { segundo) }\end{array}$} \\
\hline & & \\
\hline Buio & Mener que $0.25 \mathrm{~B}$ & Methot que 0.108 \\
\hline Moderado & $\begin{array}{l}\text { Mayor o leval que } 0.25 \mathrm{~g} r \\
\text { menor que } 0.50 \mathrm{~g}\end{array}$ & $\begin{array}{l}\text { Mayor o igual que } 0.10 \mathrm{~g} v \\
\text { menor que } 0.20 \mathrm{~g}\end{array}$ \\
\hline Moderadamente Alto & $\begin{array}{l}\text { Mayor o igual que } 050 \mathrm{~g} y \\
\text { menor que } 1.00 \mathrm{~g}\end{array}$ & $\begin{array}{l}\text { Mayor o igual que } 0.20 \mathrm{~g} \text { y } \\
\text { menor que } 0.40 \mathrm{~g}\end{array}$ \\
\hline Alto & $\begin{array}{l}\text { Mayor o igual que } 100 \mathrm{gr} \\
\text { menor que } 1.50 \mathrm{~g}\end{array}$ & $\begin{array}{l}\text { Mayor o igual que } 0.40 \mathrm{~g} \mathrm{~V} \\
\text { menor que } 0.60 \mathrm{~g}\end{array}$ \\
\hline Muy Alto & Mayer o izual que $1.50 \mathrm{~g}$ & Mayor o ifual que 0.508 \\
\hline
\end{tabular}

Nota: $\mathrm{g}=$ aceleración de le gravedad en dirección horizontal.

Fuetie. (FEMA, 2015, pús, 16, Capitulu 2). Tradacido por los astores

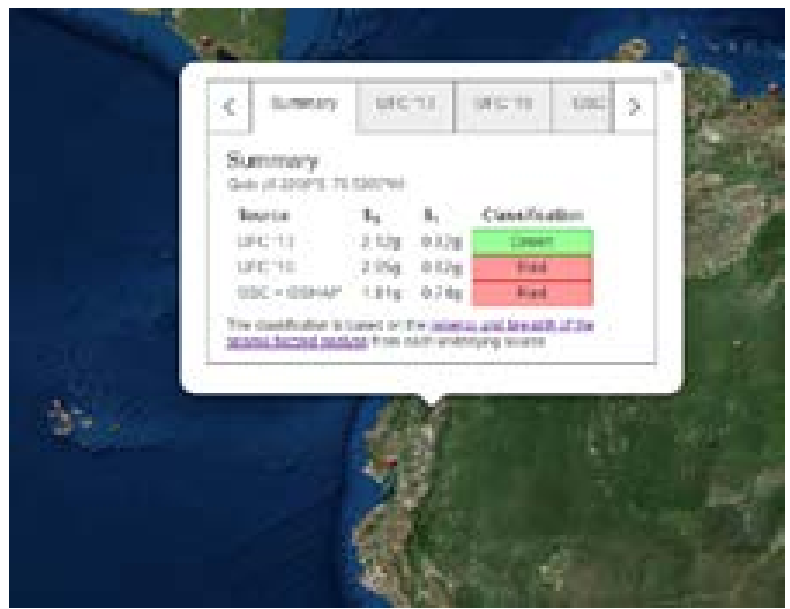

Gráfico 1. Valores para los coeficientes Ss y $\mathrm{S}_{1}$ para el sector La Armenia 1.

Fuente: (U.S. Geological Survey, 2016) 


\section{Resultados Provistos por la Evaluación RVS}

\section{Tipos de Estructuras}

Los tipos de estructuras a los que se hacen mención en este estudio son los detallados en la norma FEMA P-154.

En el sector evaluado se encontraron un total de 57 edificaciones iden- tificadas como C3 del total de las 80 edificaciones evaluadas. Esta cantidad corresponde al $71 \%$, mientras que las edificaciones $\mathrm{C} 1$ y URM son las que ocupan los siguientes lugares. En el (Gráfico 2) se pueden observar los resultados por porcentajes de edificaciones presentes en el sector.

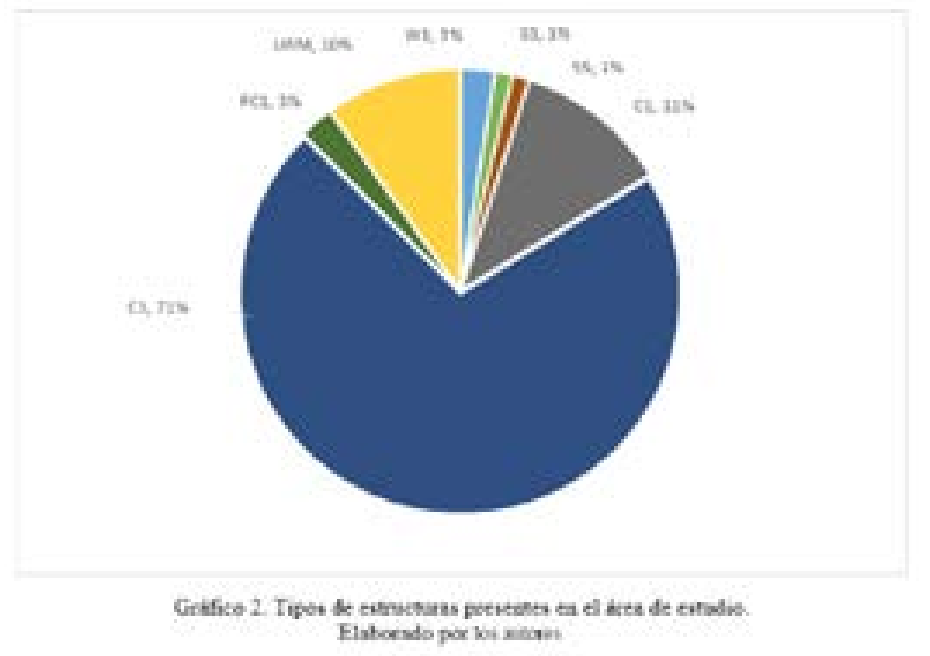

En el Ecuador la mayoría de edificaciones existentes son de hormigón armado, pues las estructuras con este material son las que mayor demanda tienen en el mercado ecuatoriano. De tal manera la mayoría de viviendas existentes son construidas con vigas y columnas de hormigón armado y con mampostería sin refuerzo estructural; este tipo de construcciones en FEMA corresponden a las C3.

Las edificaciones predominantes encontradas en la zona de evaluación fueron efectivamente las de tipo C3; al ser una zona residencial, la mayoría de viviendas tienen este estilo de construcción como se muestra en el (Gráfico 3). 


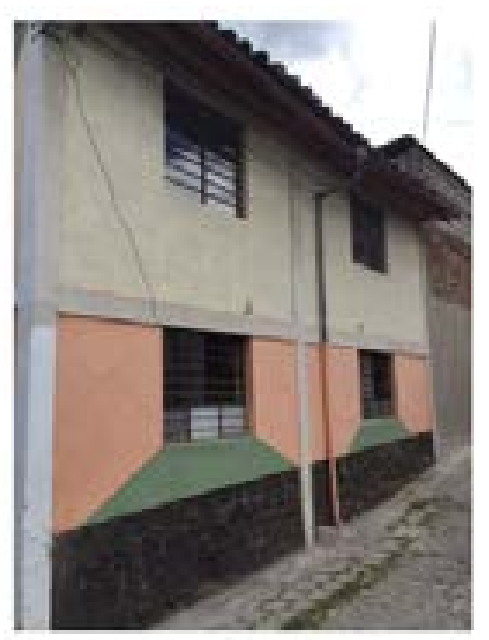

Grifico 3. Dluatraciōn de edifiesción C3.

Aunque las edificaciones C3 tienen muchas desventajas, las edificaciones URM (Edificaciones de Mampostería no Reforzada) presentan una mayor vulnerabilidad, pues carecen de un sistema estructural que resista las cargas que se producen por un movimiento sísmico. En el Ecuador todavía existen un gran número de construcciones antiguas hechas de ladrillo y adobe; como se indica en el (Gráfico 2), pues el 10\% de las edificaciones evaluadas corresponden a este tipo de estructuras. En el (Gráfico 4) se presenta un ejemplo de este tipo de edificaciones, encontrada en el sector de estudio.

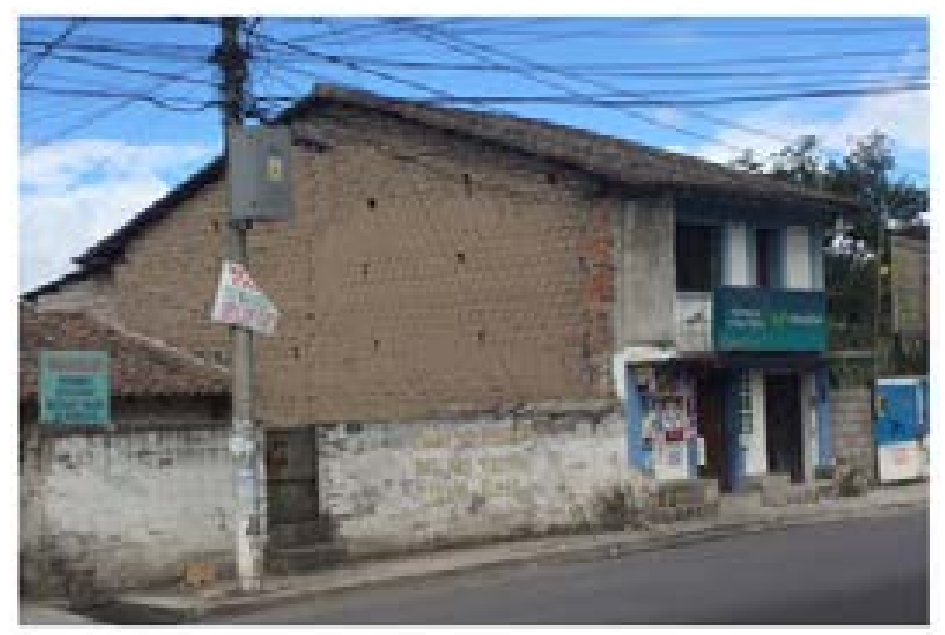

Gráfice 4, Ilustración de edificación URM. 


\section{Irregularidades}

El comportamiento de una estructura ante un movimiento sísmico se ve influenciado en gran parte por la forma de la estructura; lo ideal es que en una estructura el centro de masas coincida con su centro de rigideces para evitar que se produzcan efectos de torsión (Riesgo Sísmico, Evaluación, Rehabilitación de estructuras NEC-SE-RE, 2015). Muchas veces por motivos arquitectónicos la estructura tiene formas irregulares que producen cambios en su rigidez y resistencia, produciendo que los esfuerzos se acumulen en determinadas partes de la estructura y el riesgo de daño de la misma sea más elevado.

Las irregularidades pueden ser de dos tipos, en planta o en elevación. Para los dos tipos de irregularidades existentes se tienen diversos casos de afectación a la estructura, en el (Gráfico 5) se presentan las irregularidades encontradas en la zona de estudio.

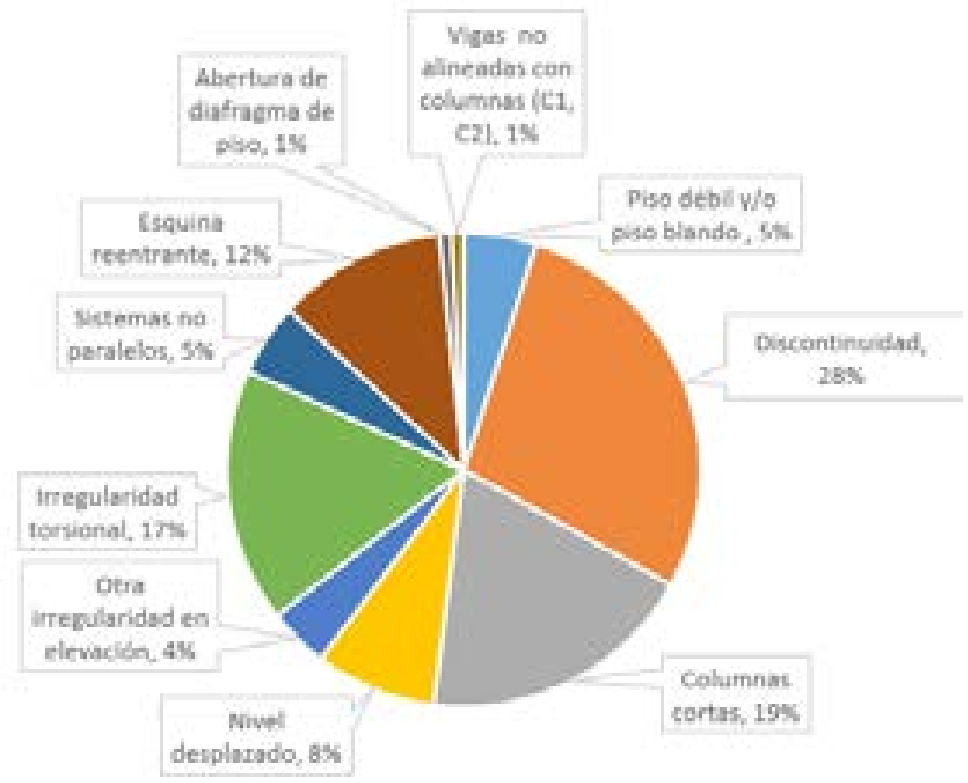

Gráfico 5. Irregularidades identificadas durante la evaluación. Elaborado por: Pablo Almagro y Xavier Paredes. 


\section{Peligros Estructurales}

Después de haber aplicado los modificadores de calificación a cada una de las estructuras según sus características constructivas, se obtuvieron los resultados de la calificación del nivel 1 y los resultados definitivos correspondientes al nivel 2. Como ya se determinó anteriormente se considera que una estructura necesita de una evaluación

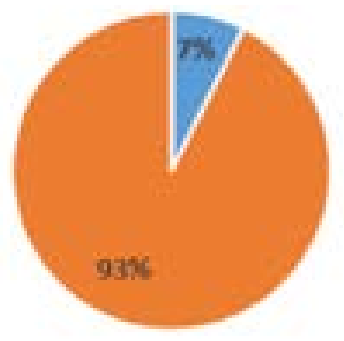

estructural cuando su puntuación es menor a 2.

Los resultados obtenidos de la evaluación indicaron que 6 edificaciones no requerían una evaluación estructural detallada y 74 edificaciones si requerían de esta evaluación, estos resultados se aprecian en el Gráfico 6 y se los puede visualizar en el Anexo 3.

\section{Gráfico 6. Resultados Nivel de Evaluación 2. Elaborado por los autores}

\section{Peligros No Estructurales}

Adicionalmente la evaluación permite determinar si se requiere una evaluación no estructural detallada. Durante la evaluación que se realizó se encontraron peligros no estructurales como chimeneas, parapetos, etc; que podían ser mitigados y no necesariamente ser evaluados.

Se necesita criterio y experiencia para determinar si un peligro no es- tructural es mitigable o necesita de una evaluación detallada, por lo que los resultados obtenidos en este estudio son subjetivos, es decir dependen del juicio de quien realice la evaluación. En el sector evaluado se determinó que 67 edificaciones no requieren de una evaluación no estructural detallada, mientras que 13 si lo requieren. Estos resultados se indican en el Gráfico 7. 

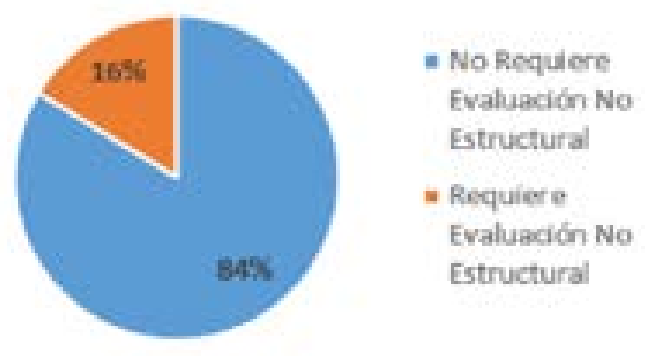

\section{Gráfico 7. Peligros no estructurales.}

Elaborado por los autores

\section{RIESGO VOLCÁNICO}

De los posibles escenarios eruptivos del volcán Cotopaxi (Samaniego, 2004) menciona que el más probable es aquel con un índice de explosividad volcánica, "V.E.I" por sus siglas en inglés, igual a 4. Evento que sería de similares características a la gran erupción acaecida el 26 de junio de 1877.

\section{Metodología de Evaluación}

Riesgo se define como la probabilidad de que ocurra un peligro y la magnitud de daño que se genere debido a la vulnerabilidad (Aneas de Castro, 2000). Por lo que para la evaluación del riesgo se debe cuantificar el mismo determinando la probabilidad y gravedad de daño que puede ocasionar el evento, en el presente estudio los eventos que se evalúan son los fenómenos volcánicos.

La evaluación de los fenómenos volcánicos que afectarán a las edificaciones del sitio en estudio se llevará a cabo con la generación de una matriz de riesgos, para cuyo fin se establece en primera instancia la siguiente fórmula, misma que cumple con lo que se ha mencionado sobre evaluación de riesgos.

La magnitud de daño que el fenómeno puede causar y la probabilidad de ocurrencia del mismo se valorarán como se indica:

$$
\begin{aligned}
& 1=\text { Baja o Ninguna } \\
& 2=\text { Mediana } \\
& 3=\text { Alta }
\end{aligned}
$$


Con los parámetros anteriormente definidos se establece el siguiente gráfico que nos ayudará a entender cómo se realizará la evaluación cualitativa del riesgo. La cual se ha elaborado a base de lo que se destaca en (Gestión de Riesgo, 2009).

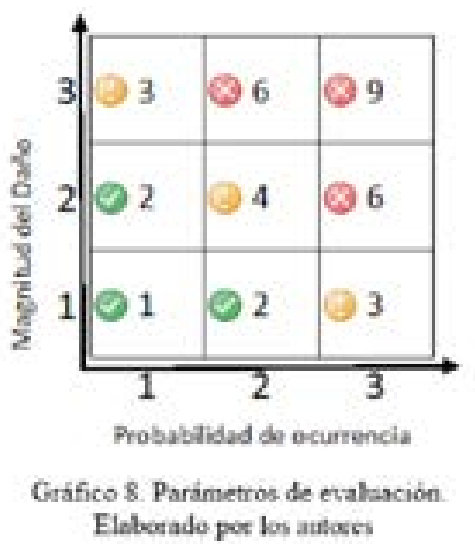

Tomando en cuenta los parámetros ya definidos para calificar la ocu- rrencia y la magnitud del daño podemos encontrar el producto de los mismos y con esto obtener la evaluación cualitativa para cada riesgo. De donde se puede identificar tres tipos de riesgo.

Bajo Riesgo $=1-2$ (Aceptable)

Medio Riesgo $=3-4$ (Advertencia) Alto Riesgo $=6-9 \bigotimes$ (Peligro inminente)

Los principales fenómenos naturales que se generarían como producto de la erupción del volcán Cotopaxi ordenados de menor a mayor peligrosidad según menciona (Andrade, y otros, 2005) son los detallados en la Tabla 2 y se los ha valorado de acuerdo con los criterios anteriormente mencionados y en función a la recopilación de datos históricos por medio de informes, narraciones de eventos pasados, estudios geológicos, entre otros. 
Tabla 2. Matriz de riesgos de los fenómenos tolcienicos del Cotopexi.

\begin{tabular}{|c|c|c|c|}
\hline Feavemese velcanico & $\begin{array}{l}\text { Magnaticud } \\
\text { del datio }\end{array}$ & $\begin{array}{l}\text { Probabilidad } \\
\text { de Ocurrencia }\end{array}$ & Kiespo \\
\hline Sismos volctricos & 1 & 1 & $O_{1}$ \\
\hline Gasen volctinicon & 1 & 2 & $\mathrm{O}_{2}$ \\
\hline Flogos de lava & 1 & 2 & \\
\hline Donos de lana & 1 & $t$ & $0_{1}$ \\
\hline Mhos pesoclasticos & 1 & 3 & $\theta_{3}$ \\
\hline Luvia de cetria y puochitos & 2 & 3 & $0_{6}$ \\
\hline Fhajo de lodo yescombres & 3 & 3 & Q9 \\
\hline Avalanchas de excoenteos & 1 & 1 & $O_{1}$ \\
\hline
\end{tabular}

Elaboesdo por las amtores

Como se observa en la matriz los fenómenos que presentan un mayor riesgo para el sector en estudio son la lluvia de ceniza y piroclastos, así como el flujo de lodo y escombros. Es así que a continuación se realiza un estudio del posible nivel de afectación que tendrían las edificaciones ante la ocurrencia de estos dos fenómenos volcánicos.

\section{Afectación Ante la Lluvia de Ceniza y Piroclastos}

Los piroclastos de gran tamaño son propensos a caer en las cercanías del cráter del volcán, por consiguiente, debido a la distancia a la que se encuentra el sector que se evalúa en esta investigación, 40 kilómetros, el estudio de la afectación del fenómeno se centrará en ver el daño que podría ocasionar la ceniza, que por acción del viento y al ser pequeña y liviana puede viajar grandes distancias.

Para realizar la metodología de evaluación, el estudio se basó en los tipos de cubiertas presentes en las edificaciones; se encontraron 5 tipos para los cuales se les asignó a cada uno su respectivo 
EVALUACIÓN TÉCNICO-VISUAL DE ESTRUCTURAS SEGÚN NEC-SE-RE EN EL SECTOR

"LA ARMENIA 1" PARA LA DETERMINACIÓN DE RIESGO ANTE FENÓMENOS NATURA\|\|$\|$ LES ESPECÍFICOS.

grado estimado de afectación. A conti- nuación, se presenta lo mencionado.

Tabla 3. Tipos de cubiertas y sus valoraciones

\section{Tipo de Cubierta Grado de Afectación Estimado}

\begin{tabular}{lll}
\hline Teja & Grave & $\bigcirc$ \\
\hline Láminas de acero delgadas & Grave & $\bigcirc$ \\
\hline Paneles de Acero & Moderado & $\bigcirc$ \\
\hline Fibrocemento & Moderado & $\bigcirc$ \\
\hline Losa de hormigón armado & Bojo & $\bigcirc$
\end{tabular}

Elaborado por los autores

Los resultados obtenidos en este nes y su tipo de cubierta se describen en análisis del número total de edificacio- la Tabla 4 y Gráfico 9.

Tabla 4. Edificaciones clasificadas por el tipo de cubierta

\begin{tabular}{lcc}
\hline \multicolumn{1}{c}{ Tipo de Cubierta } & \# Unidades & $\mathbf{\%}$ \\
\hline Teja & 26 & 32 \\
Hojas de Zinc & 6 & 8 \\
Planchas de Fibrocemento & 14 & 18 \\
Panel de Acero & 5 & 6 \\
Losa de Hormigón Armado & 29 & 36 \\
Total Edificaciones & $\mathbf{8 0}$ & $\mathbf{1 0 0}$ \\
\hline
\end{tabular}

Elaborado por los autores 


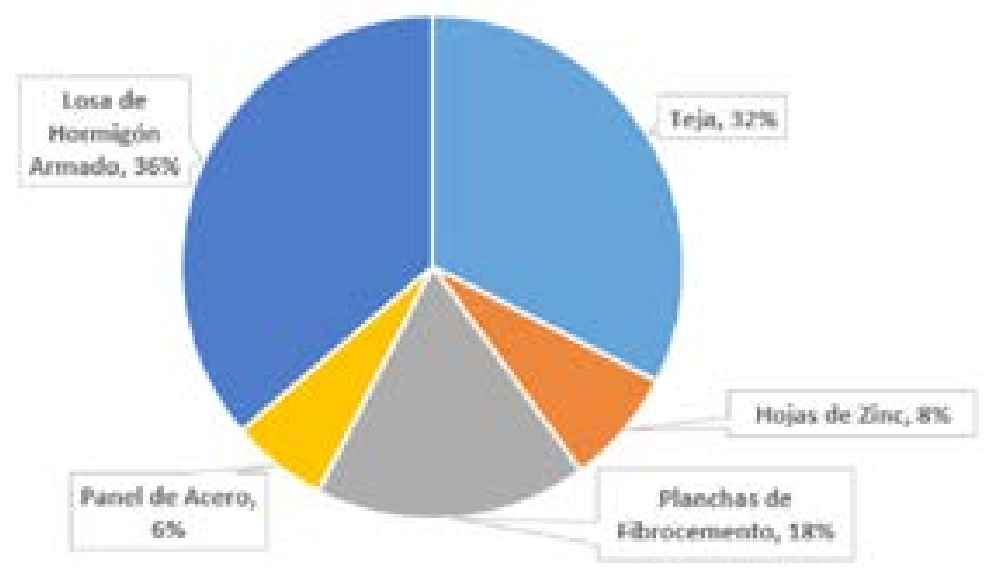

Gráfico 9. Tipo de cubiertas de edificaciones. Elaborado por los autores

En la Tabla 5 y Gráfico 10 que se caciones evaluadas. Adicionalmente se indican a continuación, se presenta la puede observar en el Anexo 4 los resulcantidad y el respectivo porcentaje del tados obtenidos en el área evaluada. grado de afectación del total de edifi-

Tabla 5. Edificaciones clasificadas según el grado de afectación en su cubierta.

\begin{tabular}{lcc}
\hline Grado de Afectación & \# Cnidades & $\%$ \\
\hline Bajo & 29 & 36 \\
Moderado & 19 & 24 \\
Grave & 32 & 40 \\
Total Edificaciones & so & 100
\end{tabular}

Elaborado por los autores 


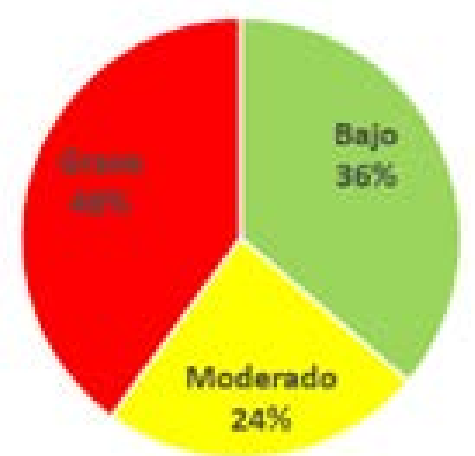

Gráfico 10. Grado de Afectación de edificaciones.

Elaborado pot los autores

\section{Estudio de Afectación de los Flujos de Lodo y Escombros}

La determinación del grado de afectación de cada una de las edificaciones se ha determinado en función de la altura de sumergimiento que tendría cada una de las estructuras si se produjese un lahar debido a la erupción del Cotopaxi.

En las cercanías al sector en estudio se encuentran tres ríos principales los cuales Ilevarían los lahares del volcán Cotopaxi, estos son los ríos Pita, San Pedro y Santa Clara. El río Santa Clara desemboca en el río San Pedro antes del sector conocido como Bocatoma; y a la altura del colegio Farina el río San Pedro se une con el río Pita. Sin embargo, en caso de una erupción los ríos Santa Clara y Pita llevarán los lahares pero unirán sus caudales antes del centro comercial San Luis Shopping. Es por esta razón que se asumió como altura más desfavorable a los flujos laháricos transportados por el río Pita en las cercanías del sector en evaluación, que es de 15 metros según lo que menciona (Aguilera \& Toulkeridis, 2005).

Tomando en cuenta este precedente se ha determinado que la altura del lahar en este sector llegaría a los 2459 metros sobre el nivel del mar; esta altura esperada de crecida se la determinó al sumar los 15 metros a la cota de fondo de río que se encuentra a los 2444 m.s.n.m. El lugar donde se determinó la cota de fondo del río fue antes del embaulamiento del mismo; a partir de este 
punto el río se encuentra embaulado debido a las recientes obras viales del sector.

Una vez que se establecieron las cotas de cada una de las edificaciones y la cota a la que puede llegar el lahar en caso de erupción del volcán Cotopaxi, se determinaron criterios para evaluar el grado de afectación que tendría una estructura, principalmente según el grado de sumergencia que tendrán cada una de las edificaciones por el paso de los flujos laháricos. Los grados de afectación estimados se describen a continuación

Tabla 6 . Alturas de Sumergencia y su grado de afectación

\begin{tabular}{cc}
\hline Tipo de Cubierta & Grado de Afectación Estimado \\
\hline O metros & Nulo \\
\hline Menor o igual a $1 \mathrm{~m}$. & Bajo \\
\hline $\begin{array}{c}\text { Desde un metro hasta } \\
\text { cubrir la planta baja. }\end{array}$ & Moderado \\
\hline $\begin{array}{c}\text { Altura del lahar mayor al } \\
\text { nivel de la planta baja. }\end{array}$ & Grave \\
\hline $\begin{array}{l}\text { Nota: En el grado de ie:eridad moderado no ie consideran las edificaciones } \\
\text { de un solo piso, pues en caso ya seria de una serendad grave }\end{array}$
\end{tabular}

\section{Elaborado por los attores}

Del estudio de afectación de las edificaciones ante flujo de lodo y escombros se ha identificado el grado de afectación para el total de estructuras, cuyos resultados se presentan en la Tabla 7 y Gráfico 11 que se indican a continuación y cuya representación en el área de evaluación se encuentra en el Anexo 5. 
EVALUACIÓN TÉCNICO-VISUAL DE ESTRUCTURAS SEGÚN NEC-SE-RE EN EL SECTOR

"LA ARMENIA 1" PARA LA DETERMINACIÓN DE RIESGO ANTE FENÓMENOS NATURA\|\|$_{\|}$LES ESPECÍFICOS.

Tabla 7. Edificaciones clasificadas según el grado de afectación.

\begin{tabular}{lcc}
\hline Grado de Afectación & i Unidades & $\%$ \\
\hline Nulo & 27 & 34 \\
\hline Bajo & 18 & 22 \\
\hline Moderado & 3 & 4 \\
\hline Grave & 32 & 40 \\
\hline Total Edificaciones & 80 & 100 \\
\hline
\end{tabular}

Elaborado por los autores

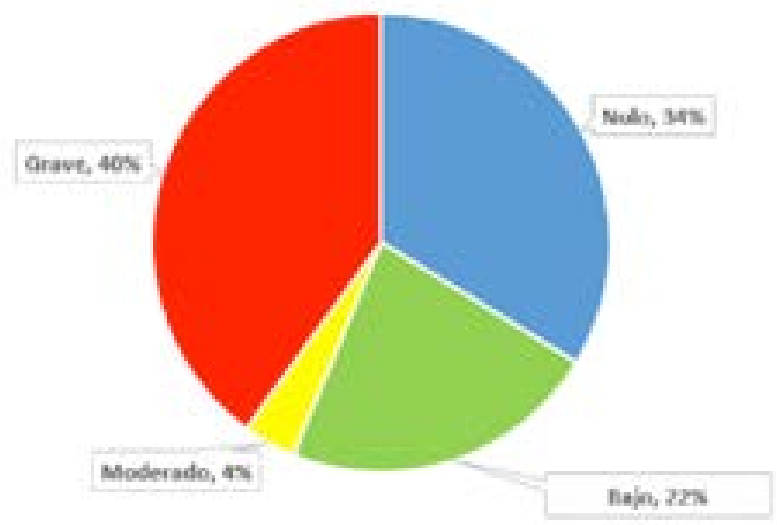

Grifice 11. Grado de Atectacion de edificacioeses.

Elaborado por los amlores 


\section{CONCLUSIONES}

Como resultado final al aplicar la metodología FEMA P-154 se obtuvo que el 93\% de las estructuras requieren de una evaluación estructural detallada, y el 16\% requiere de una evaluación no estructural detallada. El alto porcentaje de edificaciones que requieren de una evaluación estructural detallada se debe principalmente a que en el sector de estudio el $71 \%$ de las estructuras corresponden a las tipo C3, mismas que tienen una calificación inicial de 0.9 y al no haberse encontrado el modificador "Readecuaciones" en ninguna de las estructuras del sector que aumentaría la calificación inicial en +1.2 , con lo que se conseguiría llegar al 2.1 que ya cumpliría con el criterio del cut-off, el mismo que la clasificaría como una edificación que no requiere evaluación estructural detallada. El único calificador positivo para este tipo de edificación encontrado fue el de redundancia por lo que la máxima calificación que obtuvieron las estructuras de este tipo fue 1.1.
Tomando en cuenta el espesor de ceniza que podría caer sobre las cubiertas del sector en evaluación, la investigación ha concluido que las cubiertas de hormigón armado siempre y cuando se diseñen con lo que se especifique en la Norma Ecuatoriana de la Construcción no presentarían ningún problema ante una lluvia de ceniza, este porcentaje de edificaciones para el sector es de 36\%.

El estudio realizado en esta investigación de las edificaciones afectadas por los flujos de lodo y escombros ha concluido que el sector La Armenia 1 tiene una zona de afectación semejante a la que ha determinado la Secretaría de Gestión de Riegos. Específicamente la diferencia que se puede establecer es en aquellas edificaciones que en el estudio se han identificado con un grado de afectación bajo o nulo, las mismas que en el mapa de amenazas del volcán Cotopaxi de la Secretaría de Gestión de Riesgos varían en cuanto a si son afectadas o no.

\section{RECOMENDACIONES}

Es importante mencionar que el estudio que se ha realizado en cuanto a la evaluación de las estructuras ante eventos sísmicos, la matriz de riesgos para eventos volcánicos, así como el estudio de los fenómenos volcánicos: lluvia de ceniza y piroclastos, y de flujo de lodos y escombros, tiene su grado de 
incertidumbre y no deben ser tomados como valores definitivos, sino más bien como un esfuerzo por determinar el posible grado de afectación de las edificaciones ante los fenómeno antes mencionados, por lo que posteriores y más minuciosos estudios de las edificaciones del sector podrían no estar de acuerdo completamente con lo que se describe en esta investigación.
De la información que se ha recopilado se evidencia que en el Distrito Metropolitano de Quito se pueden observar varias construcciones edificadas en zonas de riego por lo que se recomienda que las entidades públicas tengan un mayor control en cuanto a la planificación del desarrollo urbano, uso del suelo y un control adecuado con respecto a la ejecución de las estructuras. 


\section{BIBLIOGRAFÍA}

Aguilera, E., \& Toulkeridis, T. (2005). El Volcán Cotopaxi, una amenaza que acecha. Quito.

Andrade, D., Hall, M., Mothes, P., Troncoso, L., Eissen, J.-P., Samaniego, P., ... Yepes, H. (noviembre de 2005). Los peligros volcánicos asociados con el Cotopaxi. Quito, Ecuador: Corporación Editora Nacional.

Aneas de Castro, S. (2000). Riesgos y peligros: una visión desde la Geografía. Scipta Nova (en línea) (60). Obtenido de http://www. ub.edu/geocrit/sn-60.htm

Chamorro, D. (14 de agosto de 2015). El Instituto Geofísico reportó dos explosiones en el volcán Cotopaxi; ceniza cae en el sur de Quito. El Comercio. Recuperado el 19 de julio de 2016, de http:// www.elcomercio.com/actuali$\mathrm{dad} /$ ministerio-ambiente-explosiones-volcan-cotopaxi.html

Cuatro temblores sacudieron a Quito. (12 de agosto de 2015). El Comercio. Recuperado el 19 de julio de 2016, de http://www. elcomercio.com/actualidad/ temblor-sacudio-quito.html
FEMA. (2015). Rapid Visual Screening of Buildings for Potencial Seismic Hazards: A Handbook (Tercera ed.).

Gestión de Riesgo. (2009). Recuperado el 15 de agosto de 2016, de https://protejete.wordpress.com/ gdr_principal/matriz_riesgo/

IGEPN. (17 de abril de 2016). INFORMESÍsMICO ESPECIAL N. 13 - 2016. Recuperado el 19 de julio de 2016, de http://www.igepn.edu.ec/ servicios/noticias/1317-informe-sismico-especial-n-13-2016 Peligro Sísmico Diseño Sismo Resistente NEC-SE-DS. (2015).

Riesgo Sísmico, Evaluación, Rehabilitación de estructuras NEC-SE-RE. (2015). Secretaría de Gestión de Riesgos. (s.f). Mapa de amenaza volcán Cotopaxi. Obtenido de https://www. google.com/maps/d/viewer?mid=1 YiUOIQopou41BghFsIEuFGxngXE 
EVALUACIÓN TÉCNICO-VISUAL DE ESTRUCTURAS SEGÚN NEC-SE-RE EN EL SECTOR

"LA ARMENIA 1" PARA LA DETERMINACIÓN DE RIESGO ANTE FENÓMENOS NATURA\|\|$_{\|}$LES ESPECÍFICOS.

\section{ANEXo 1. "LA ARMENia 1", ÁrEA de EsTUdio}

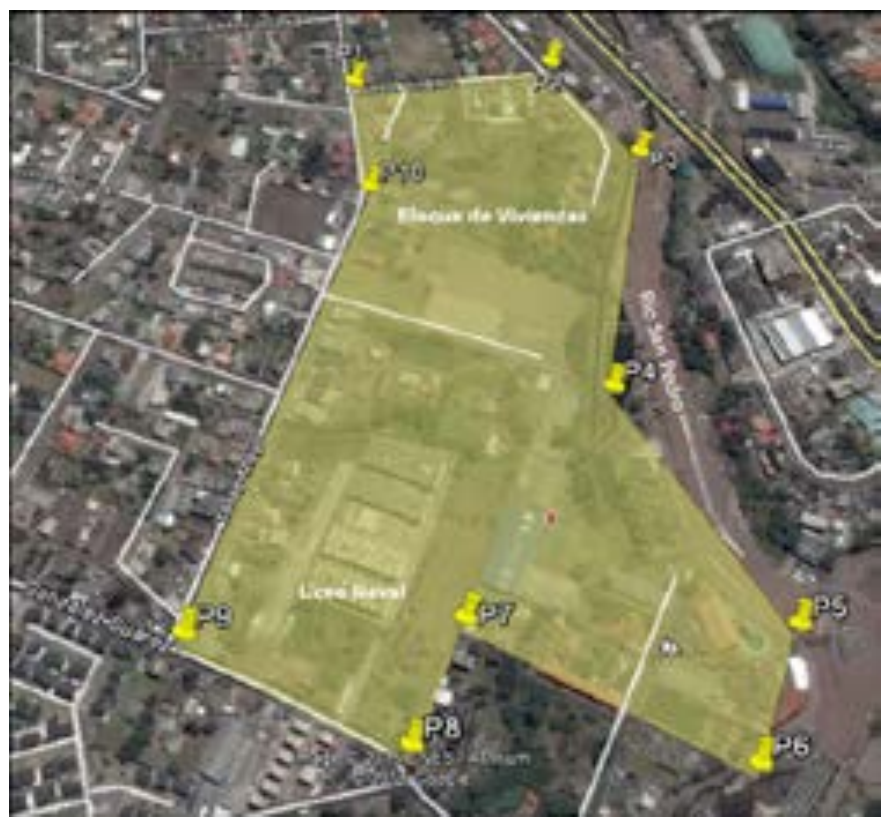

Área a Evaluarse. Adaptado de: Google Earth.

Elaborado por los autores

\begin{tabular}{|c|c|c|}
\hline \multicolumn{3}{|c|}{ 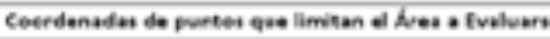 } \\
\hline Pte & Latifed & Lougitud \\
\hline PI & $0^{*} 17^{-39.57 \% 5}$ & $75^{\circ} 27 \cdot 35.29 \cdot \mathrm{W}$ \\
\hline$P 2$ & $0 \cdot 17 \cdot 35.71 \% \mathrm{~s}$ & $75 \cdot 27 \cdot 4937 \times W$ \\
\hline P3 & $0^{\prime} 17742.15 \mathrm{~s}$ & $75^{\circ} 27^{\circ} 4696^{\prime} \mathrm{W}$ \\
\hline P4 & $0.17 \% 49.97 \mathrm{~s}$ & $75^{\circ} 27.48 .40^{\prime} \mathrm{W}$ \\
\hline P5 & $0.17^{-3661^{-2} \mathrm{~s}}$ & $75 \cdot 27 \cdot 4.48 * \mathrm{~W}$ \\
\hline P6 & $0.17 \times 99.9475$ & $75^{\circ} 27 \cdot 4590^{\prime} \mathrm{W}$ \\
\hline P7 & $0.17 \div 56.42 \% \mathrm{~s}$ & $75^{\circ} 27^{\prime} 5251^{\prime \prime} \mathrm{W}$ \\
\hline P8 & $0^{*} 17^{2} 59.47^{*} s$ & $75^{\circ} 27^{\circ} 53.86^{\circ} \mathrm{W}$ \\
\hline P9 & $0.17 \times 8.827 \mathrm{~s}$ & $75^{*} 27^{*} 39.18^{\prime} \mathrm{W}$ \\
\hline P10 & $0^{2} 17^{\prime} 43.49^{\prime \prime} \mathrm{s}$ & $75^{\circ} 27^{\circ} 4.81^{\prime \prime W}$ \\
\hline
\end{tabular}

\begin{tabular}{|c|c|}
\hline \multicolumn{2}{|c|}{ Liederes del perimetro del Área a Evaluarse } \\
\hline PI-P2 & Calle Jose Villalba \\
\hline P1-P10-P9 & Calle 18 de Mayo \\
\hline P7-P8 & Cale Gouzales Sasirez \\
\hline P5-P7 & Pasaje entrada a Liceo Naval \\
\hline P7-P6-P5 & Linderos Liceo Naval \\
\hline P5-P4-P3 & Borde de rio Sin Pedro \\
\hline P3-P2 & Calle Jose Jebarvea \\
\hline
\end{tabular}

Perímetro del área y coordenadas de la misma.

Elaborado por los autores 


\section{ANEXO 2. FORMULARIO FEMA P-154, REGIÓN SÍSMICA MUY ALTAANEXO 2 - FORMULARIO FEMA P-154, REGIÓN SÍSMICA MUY ALTA}

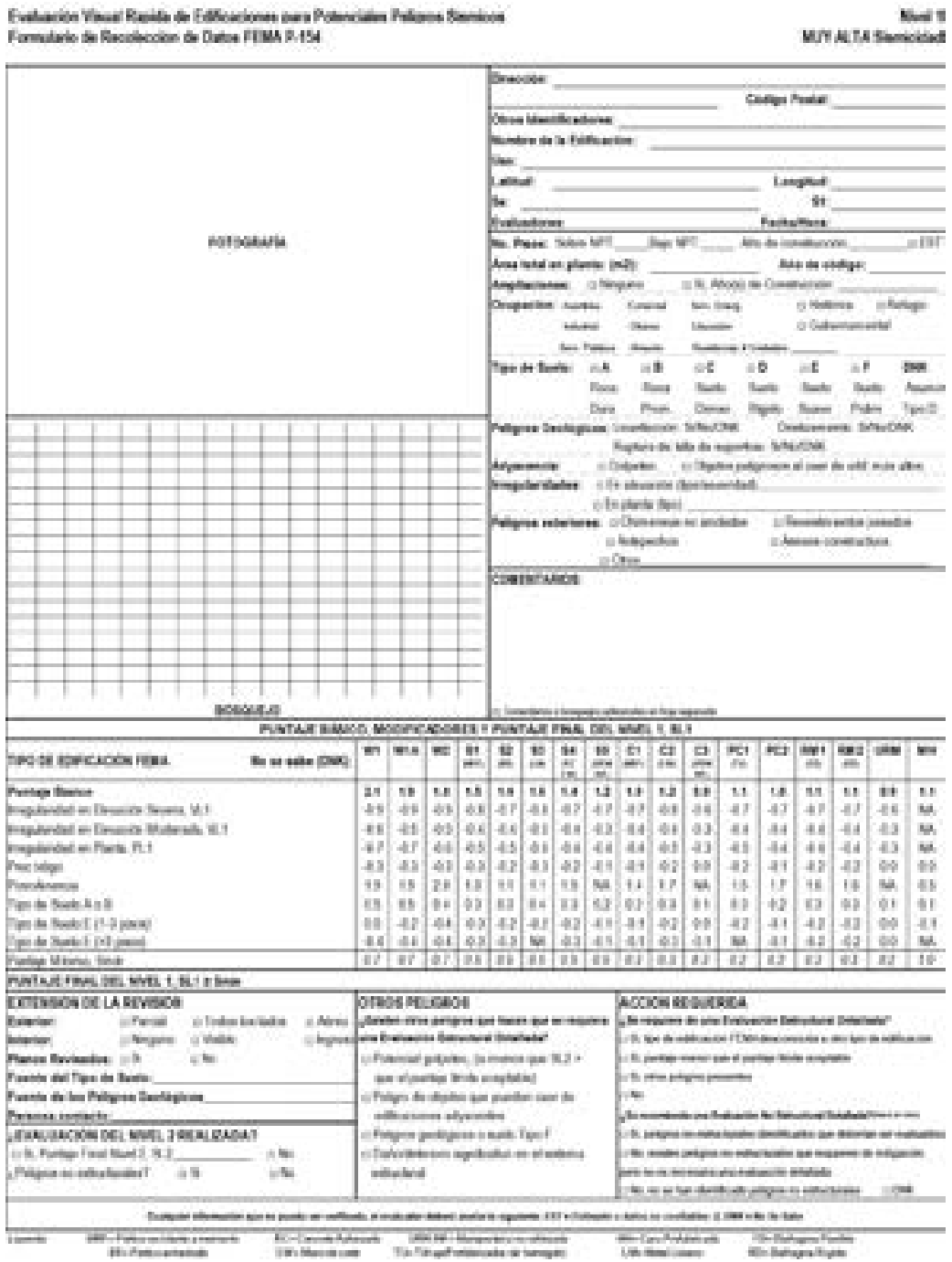


La evaluación de Nivel 2 debe ser realizada por un ingeniero civil o estructural, arquitectos o estudiantes graduados que tengan experiencia en la evaluación o diseño sismico de estructuras. Nombre de la edificación: Puntaje Final Nivel 1: SL1=

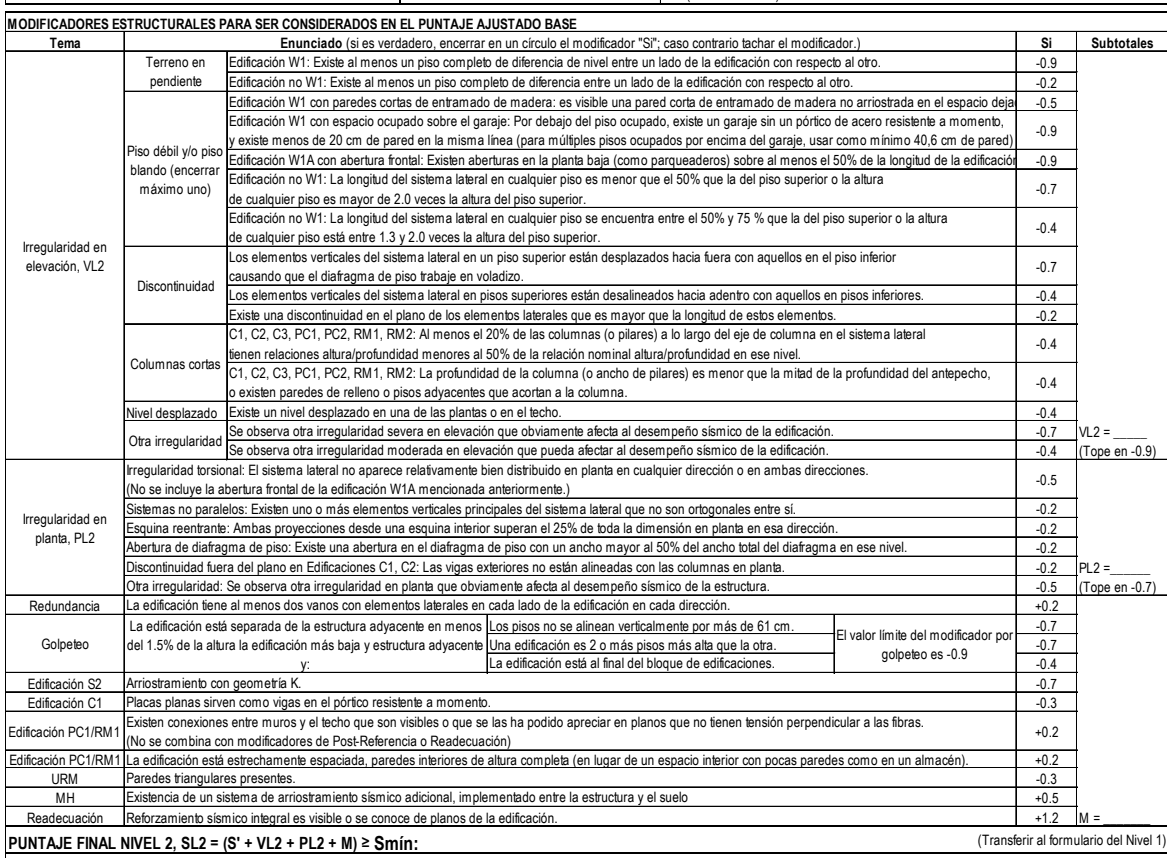

Si ha marcado "Si", describir la condición en la sección comentarios en la parte inferior e indicar en el formulario del Nivel 1 que una evaluación detallada es requerida independiente del puntaje de la edificación. PELIGROS NO ESTRUCTURALES EVIDENTES:

\begin{tabular}{|c|c|c|c|c|}
\hline Ubicación & Aspecto (Chequear "Si o "No") & $\mathrm{Si}$ & No & Comentario \\
\hline \multirow{7}{*}{ Exterior } & Existe un antepecho de mampostería no reforzada no arriostrada o una chimenea de mamposteria no reforzada & & & \\
\hline & Existen revestimientos pesados & & & \\
\hline & Existe una cubierta pesada sobre puertas de salida o pasillos peatonales que parecen estar soportados de manera inadecuada. & & & \\
\hline & Existe un anexo de mampostería no reforzada sobre puertas de salida o pasillos peatonales. & & & \\
\hline & Existe un letrero colocado en la edificación que indica que hay materiales peligrosos presentes & & & \\
\hline & Existe una edificación adyacente más alta con una pared URM no anclada o un antepecho URM no arriostrados o chimenea. & & & \\
\hline & Otro peligro exterior observable no estructural que pueda caer. & & & \\
\hline \multirow[b]{2}{*}{ Interior } & Existen tejas de arcilla o tabiques de ladrillo en gradas o corredores de salida. & & & \\
\hline & Otro peligro interior observable no estructural que pueda caer. & & & \\
\hline \multicolumn{5}{|c|}{ Desempeño Sismico No Estructural Estimado (Chequear el recuadro apropiado y transferirlo a las conclusiones del formulario del Nivel 1) } \\
\hline & \multicolumn{4}{|c|}{$\square$ Peligros potenciales no estructurales con una amenaza significativa a la seguridad de vida de los ocupantes $\rightarrow$ Evaluación No Estructural Detallada recomendada } \\
\hline & \multicolumn{4}{|c|}{$\square$ Peligros no estructurales identificados con una amenaza significativa a la seguridad de vida de los ocupantes $\rightarrow$ Pero no requiere una Evaluación No Estructural Detallada } \\
\hline & \multicolumn{4}{|c|}{$\square$ Peligros no estructurales menores o inexistentes que amenacen a la seguridad de vida de los ocupantes $\rightarrow$ No se requiere Evaluación № Estructural Detallada } \\
\hline
\end{tabular}

Comentarios: 


\section{ANEXO 3. RESULTADOS DE ESTRUCTURAS QUE REQUIEREN Y NO REQUIEREN DE UNA EVALUACIÓN ESTRUCTURAL DETA- LLADA.}

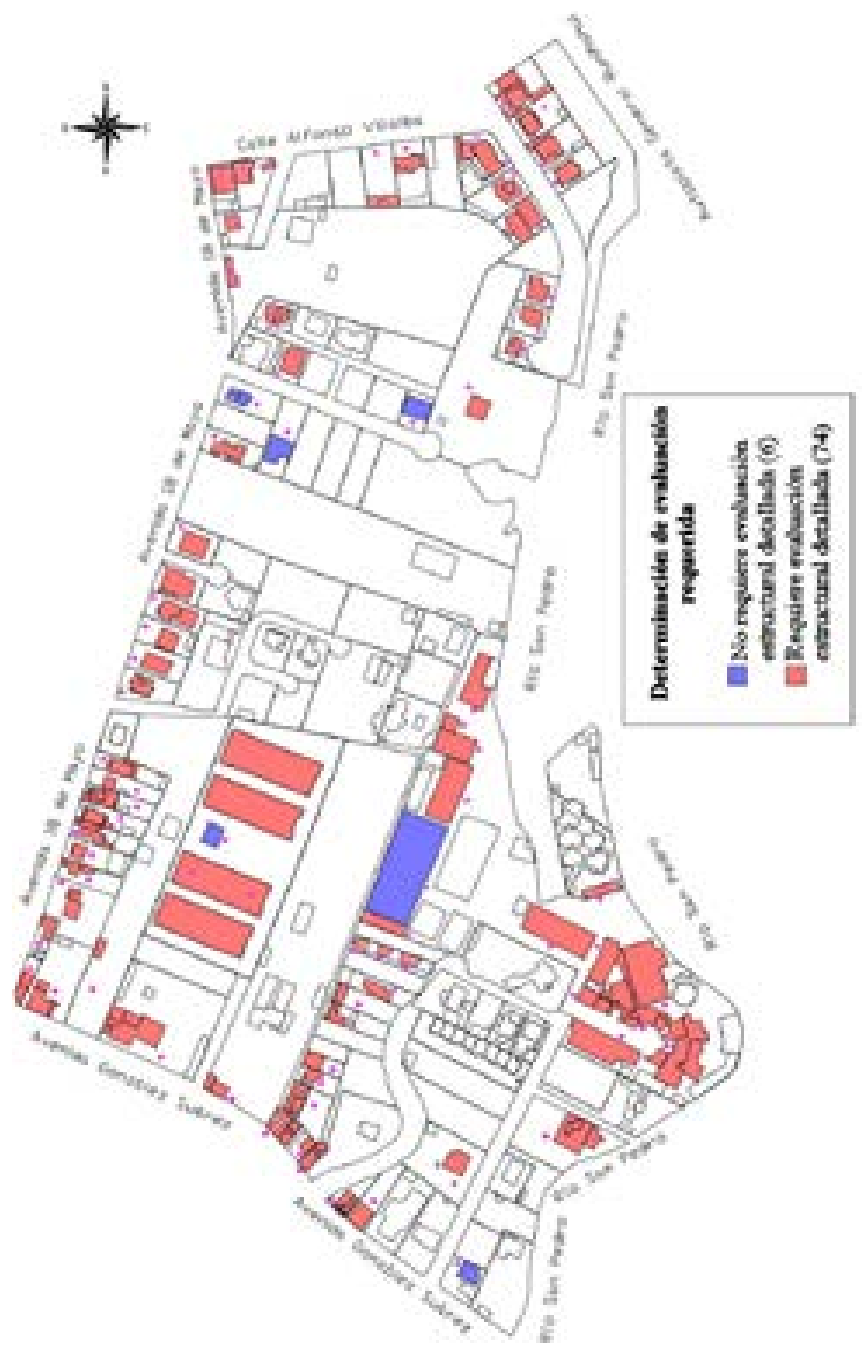




\section{ANEXO 4. RESULTADOS DEL GRADO DE AFECTACIÓN POR CAÍdA DE CENIZA Y PIROCLASTOS}

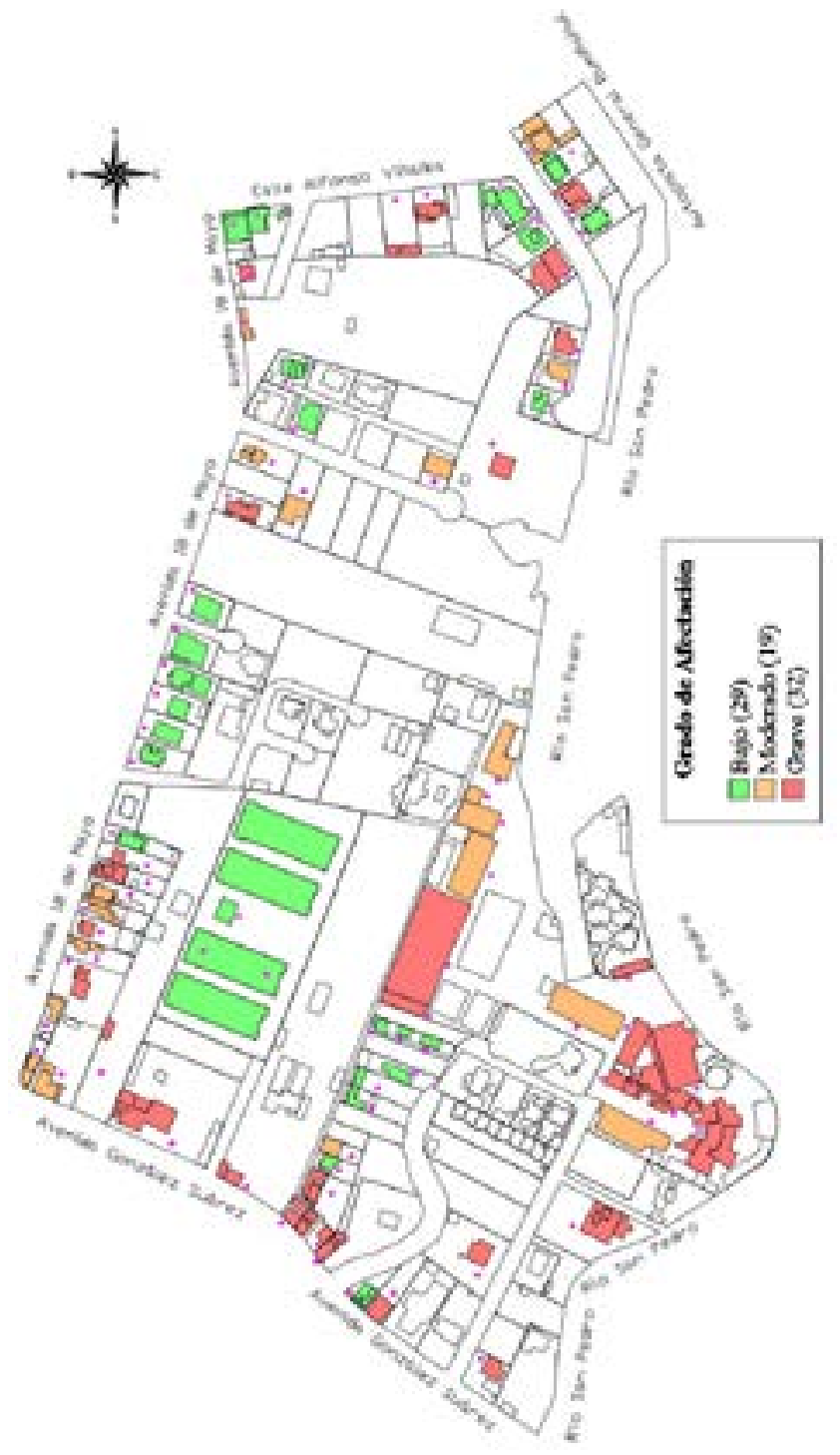




\section{ANEXO 5. RESULTADOS DEL GRADO DE AFECTACIÓN DE LAS ESTRUCTURAS ANTE LOS FLUJOS DE LODO Y ESCOMBROS}

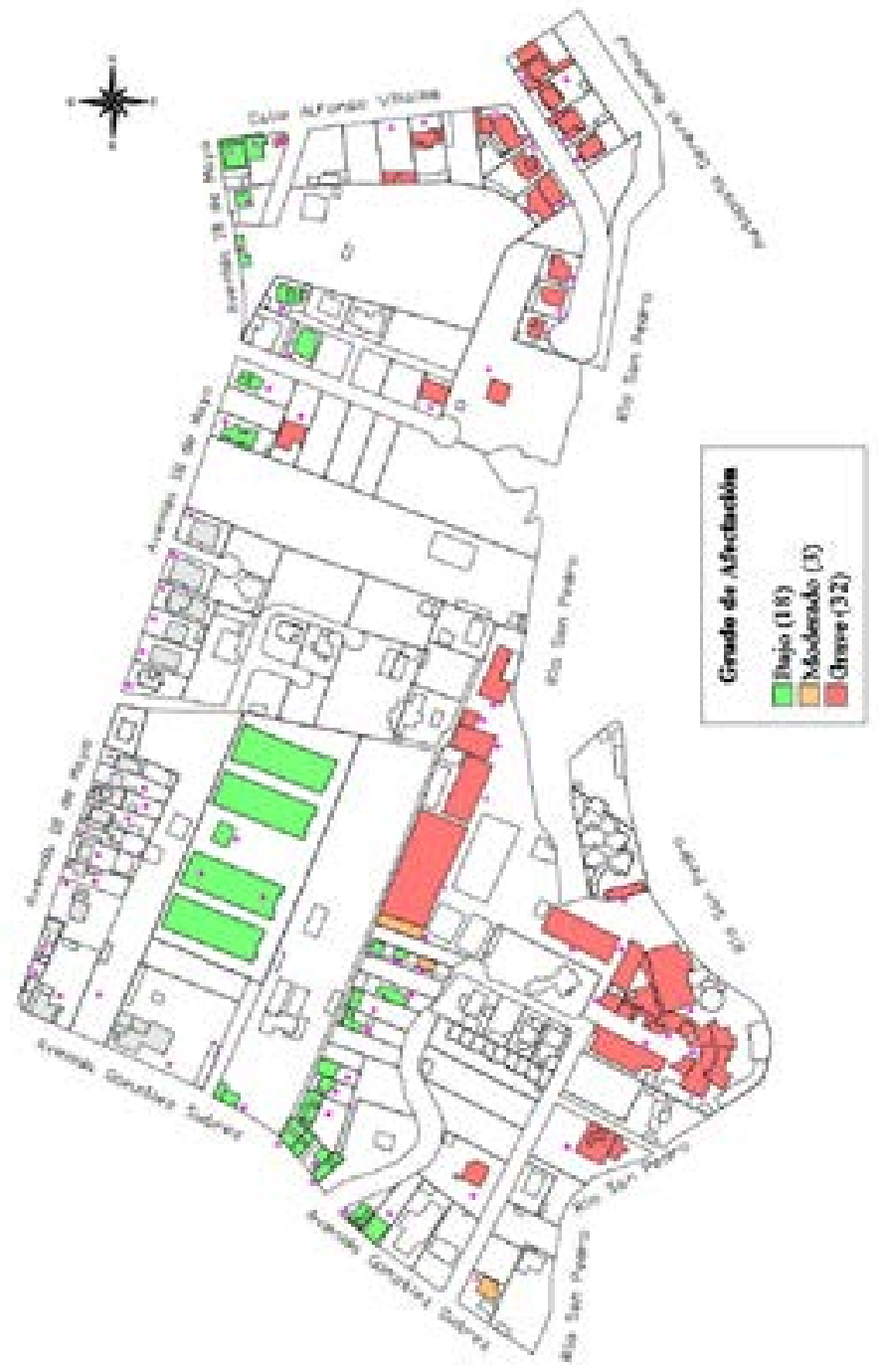


\|\|$\| 140$ 